\title{
Dimensionamento de pessoal de enfermagem e grau de dependência do paciente em um hospital universitário
}

Nursing personnel dimensioning and the dependence level of the patient in a school hospital

Dimensionamiento del personal de enfermería y el grado de dependencia de los pacientes en un hospital universitario

Gislaine Pinn Gil ${ }^{\mathrm{I}}$, Dagmar Willamowius Vituri ${ }^{\mathrm{II}}$, Maria do Carmo Lourenço Haddad ${ }^{\mathrm{III}}$, Marli Terezinha Oliveira Vannuchi ${ }^{\mathrm{IV}}$, Fernanda Novaes Morenov

\section{RESUMO}

Os objetivos do estudo foram analisar o dimensionamento de pessoal de enfermagem em hospital universitário comparando-o com o grau de dependência de pacientes atendidos em 1998 e 2008 e comparar o envelhecimento da força de trabalho da enfermagem no mesmo período. Em 2009, realizou-se um estudo comparativo, descritivo, exploratório e retrospectivo por meio de dados secundários referentes às unidades de internação médico-cirúrgicas da instituição envolvida. Os resultados apontaram que o dimensionamento de pessoal de enfermagem permaneceu defasado no decorrer de dez anos; aumento do grau de dependência dos pacientes em relação à enfermagem e envelhecimento funcional e cronológico da força de trabalho em mais da metade da população estudada. Concluiu-se que o quadro de pessoal de enfermagem não acompanhou o aumento da complexidade assistencial demandada pelos pacientes, o que é agravado pela capacidade para o trabalho reduzida dos trabalhadores.

Descritores: Downsizing organizacional; Determinação de necessidades de cuidados de Saúde; Enfermagem.

\section{ABSTRACT}

The study aimed to analyze the nursing personnel dimensioning in a school hospital compared it to the dependence level of patients admitted in 1998 and 2008 and to compare it to the aging nursing workforce in the same period. In 2009, a comparative, exploratory, descriptive and retrospective study was conducted using secondary data from medical-surgical inpatient care units of a school hospital. Results demonstrated that nursing staff dimensioning is unbalanced in the course of ten years; increasing of the patients' dependence degree level related to the nursing staff and its functional and chronological aging, more than half of the studied nurses group. It was concluded that the nursing staff did not follow the increasing complexity of care demanded by patients, which is aggravated by reduced working capacity of employees.

Descriptors: Personnel dimensioning; Needs assessment; Nursing.

\section{RESUMEN}

Los objetivos del estudio fueron analizar el dimensionamiento del personal de enfermería en un hospital-escuela en comparación con el grado de dependencia de los pacientes tratados en 1998 y 2008, y comparar el envejecimiento de la fuerza de trabajo de enfermería en el mismo período. En 2009, se realizó un estudio comparativo, descriptivo, exploratorio y retrospectivo por medio de datos secundarios referentes a las unidades médico-quirúrgicas de internación hospitalaria. Los resultados mostraron que el dimensionamiento del personal de enfermería sigue desequilibrado en el transcurso de diez años; aumento del grado de dependencia de los pacientes en relación a la enfermería y el envejecimiento funcional y cronológico de la fuerza de trabajo en más de la mitad de la población estudiada. Se concluyó que el personal de enfermería no acompañó el aumento de la complejidad de la atención demandado por los pacientes, que es agravado por la capacidad de trabajo reducida de los trabajadores.

Descriptores: Redicción de personal; Evaluación de necesidades; Enfermería.

\footnotetext{
I Enfermeira, Discente do Programa de Pós-Graduação em Enfermagem Fundamental, nível Mestrado, Escola de Enfermagem de Ribeirão Preto (EERP), Universidade de São Paulo (USP). Enfermeira do Hospital Zona Sul de Londrina. Londrina, PR, Brasil. E-mail: lainepinn@gmail.com.

II Enfermeira, Discente do Programa de Pós-Graduação em Enfermagem Fundamental, nível Doutorado, EERP, USP. Enfermeira do Hospital Universitário de Londrina, Universidade Estadual de Londrina (UEL) Londrina, PR, Brasil. E-mail: dagmar@uel.br.

III Enfermeira, Doutora em Enfermagem. Professor Associado, UEL. Londrina, PR, Brasil. E-mail: haddad@sercomtel.com.br.

IV Enfermeira, Doutora em Saúde Pública. Professor Associado, UEL. Londrina, PR, Brasil. E-mail: vannuchi@sercomtel.com.br.

$\checkmark$ Enfermeira. Discente do Programa de Pós-Graduação em Enfermagem, nível Mestrado, UEL. Londrina, PR, Brasil. E-mail: fernandanovaes@pop.com.br.
} 


\section{INTRODUÇÃO}

O dimensionamento de pessoal de enfermagem tem sido foco de atenção das instituições de saúde por intervir, diretamente, na eficácia, na qualidade e no custo da assistência. A temática também é motivo de conflitos, pois, se por um lado há a necessidade crescente de aumentar a oferta de serviços de saúde, por outro, existe a racionalização de custos gerada pela falta de recursos financeiros na área, colocando em questão o quadro de recursos humanos de enfermagem que, geralmente, representa o maior quantitativo e, consequentemente, o maior gasto orçamentário com pessoal nas organizações de saúde ${ }^{(1)}$.

Soma-se ainda à problemática da racionalização de recursos, a demanda por atendimento da clientela que, com necessidades cada vez mais especializadas e complexas, tem imprimido sobrecarga de trabalho aos integrantes da equipe de enfermagem, sobrecarga esta que acaba por influenciar e dificultar a implementação de qualquer medida que favoreça a qualidade da assistência $\operatorname{prestada}^{(1)}$.

O dimensionamento de pessoal de enfermagem pode ser definido como "um processo sistemático que fundamenta o planejamento e a avaliação do quantitativo e qualitativo do pessoal de enfermagem necessário para prover os cuidados de enfermagem "(1).

O Conselho Federal de Enfermagem (COFEN) estabelece parâmetros para o dimensionamento de pessoal de enfermagem nas instituições de saúde, com base na aplicação de um Sistema de Classificação de Pacientes, como critério para se estabelecer o perfil de cada paciente internado nas unidades hospitalares, as horas mínimas de assistência e a distribuição dos profissionais para cada tipo de cuidado $^{(2)}$. Nesta lógica, cabe ao enfermeiro o gerenciamento do seu quadro de pessoal segundo a necessidade de assistência requerida pelos pacientes, conforme a classificação de sua dependência em relação aos cuidados de enfermagem.

O enfermeiro assume uma posição de articulador de ações de enfermagem nas instituições de saúde, cabendo a ele discutir e participar ativamente na definição da política de recursos humanos da instituição, estabelecendo estratégias que subsidiem a prática da enfermagem e parâmetros que permitam um dimensionamento de pessoal, sob o enfoque quantitativo e qualitativo, capaz de atender às necessidades de assistência da clientela ${ }^{(3)}$.

Esse contexto evidencia a importância dos enfermeiros buscarem instrumentos que possibilitem um melhor gerenciamento dos recursos sob sua responsabilidade, dando atenção especial aos recursos humanos por sua relevância para a qualidade do cuidado $^{(4)}$.

Uma das ferramentas utilizadas para a gestão de pessoal é o Sistema de Classificação de Pacientes (SCP), utilizado para categorizar pacientes de acordo com a quantidade de cuidado de enfermagem requerida, ou seja, baseado no grau de complexidade de assistência de enfermagem ${ }^{(5)}$. Em outras palavras, a complexidade relaciona o grau de necessidade do paciente, por meio do volume e da intensidade de consumo de recursos, durante a assistência ${ }^{(6)}$.

Portanto, o SCP é instrumento que pode ser utilizado para subsidiar o enfermeiro a justificar necessidades adicionais de recursos humanos, diante das inadequações do quantitativo da força de trabalho ou acréscimo no volume de trabalho na unidade, além de nortear decisões relacionadas ao recrutamento e seleção de pessoal de enfermagem ${ }^{(6)}$.

No entanto, os dados obtidos no processo de classificação não são utilizados apenas para subsidiar o dimensionamento de recursos humanos, mas também na organização e melhoria da qualidade do serviço de enfermagem, distribuição de atividades entre os membros da equipe, alocação de recursos materiais, planejamento de planta física do serviço e determinação dos custos da assistência ${ }^{(7)}$.

Neste contexto, o acompanhamento da complexidade assistencial durante o período de internação permite identificar a carga de trabalho da equipe de enfermagem e o tempo despendido no cuidado, contribuindo para o planejamento mais racional das ações a serem implementadas junto aos pacientes ${ }^{(6)}$.

Nos últimos anos, surgiram várias propostas de Sistemas de Classificação de Pacientes que permitem evidenciar o grau de dependência do paciente em relação à enfermagem, e podem ser utilizadas como base para o cálculo de dimensionamento de pessoal.

Os Sistemas de Classificação de Pacientes abordam diferentes critérios de avaliação que vão desde o uso de indicadores com base nas necessidades humanas básicas, dependência em relação à assistência ou tipo de atendimento realizado. Portanto, utilizando um método que melhor se adapte e traduza a realidade de cada instituição, a classificação possibilita a identificação do paciente em relação ao nível de dependência da equipe de enfermagem. 
Com a organização da rede dos serviços de saúde, aumento da quantidade de instituições terciárias referenciadas e o crescente envelhecimento da população, acredita-se que o perfil dos clientes dos hospitais terciários está se modificando, resultando em maior demanda por procedimentos especializados para o atendimento de pacientes mais graves e dependentes. Isto culmina com o aumento do número de horas assistenciais necessárias por paciente, que se traduz em um quantitativo maior de profissionais qualificados e capacitados.

No cotidiano da prática profissional, observa-se que o trabalhador de enfermagem hoje se vê obrigado a prestar assistência a pacientes graves e dependentes fora de unidades de cuidados intensivos, em razão do número de leitos em Unidade de Terapia Intensiva (UTI) ser insuficiente para a demanda instalada. Estas unidades de internação geralmente não possuem o aparato e a infraestrutura de uma unidade de terapia intensiva e, muitas vezes, o trabalhador não está tecnicamente preparado para prestar cuidados a esse perfil de paciente, o que resulta em sobrecarga de trabalho e estresse ocupacional, que podem trazer consequências à sua saúde, além de prejuízos à instituição e a clientela.

Diante da relevância da questão do dimensionamento de pessoal em enfermagem para a qualidade da assistência e para a segurança do paciente, e da presumida alteração no perfil epidemiológico da clientela atendida em um hospital público de ensino do Estado do Paraná, é que se propôs a realização desse estudo, no sentido de analisar o quantitativo da força de trabalho da equipe de enfermagem, comparando-o com o grau de dependência dos pacientes atendidos.

Desta forma, o objetivo deste trabalho foi analisar o dimensionamento de pessoal de enfermagem em Hospital Universitário e comparar com o grau de dependência de pacientes atendidos em 1998 e 2008. Além disso, pretende-se também comparar o envelhecimento da força de trabalho da enfermagem no referido período.

\section{METODOLOGIA}

Estudo comparativo do tipo descritivo exploratório retrospectivo no Hospital Universitário Regional do Norte do Paraná (HURNP). A instituição mantém convênio exclusivo com o Sistema Único de Saúde (SUS) e é o único hospital público de grande porte do Norte do Paraná, sendo considerado o terceiro maior hospital da região Sul do País, com capacidade de internação de 333 leitos, nas diversas especialidades médicas e cirúrgicas. Atualmente, funciona com o sistema de atendimento referenciado, que abrange a $17^{\circ}$ Regional de Saúde do Paraná. Além de sua atuação no campo assistencial, são desenvolvidas atividades nos cursos de graduação e pósgraduação para estudantes e profissionais de Enfermagem, Medicina e outros cursos da área da saúde.

Para esse estudo foram utilizados dados secundários gerados pela Assessoria de Controle da Qualidade da Assistência de Enfermagem (ACQAE) do hospital. Essa assessoria foi implantada em 1998 com os objetivos de monitorar os processos de trabalho e a qualidade da assistência de enfermagem prestada, garantir o direito ao usuário de ter uma assistência de enfermagem segura e direcionar a educação continuada de acordo com as necessidades de aperfeiçoamento da equipe.

Os dados foram coletados no mês de dezembro de 2009. As informações referentes ao dimensionamento de pessoal de enfermagem foram coletadas no banco de dados da Assessoria de Planejamento e Controle, e na $A C Q A E$, os dados relativos à classificação de dependência dos pacientes internados nas unidades de internação médico - cirúrgica para pacientes adultos (Unidade Masculina e Unidade Feminina), ambos referentes aos anos de 1998 e 2008.

Nas Unidades Masculina e Feminina são internados pacientes dos respectivos sexos com idade superior a 12 anos, com diagnósticos clínicos e cirúrgicos. A Unidade Masculina está localizada em uma construção antiga com várias adaptações, de modelo pavilhonar, onde os pacientes estão distribuídos em enfermarias de seis leitos. Os banheiros estão centralizados no corredor, bem como o posto de enfermagem. Uma enfermaria de isolamento fica localizada na extremidade do setor. Anexada gerencialmente e estruturalmente à unidade, encontra-se a ala de Tisiologia que é destinada exclusivamente a pacientes portadores de doenças pulmonares.

A Unidade Feminina apresenta uma estrutura física mais adequada de acordo com a normatização estrutural, tendo sido reformada no ano de 2008. As pacientes são distribuídas em enfermarias de três a quatro leitos, com banheiros privativos por enfermaria, duas enfermarias destinadas para isolamento, dois postos de enfermagem centralizados e sala para realização de pequenos procedimentos.

O Sistema da Classificação de Pacientes utilizado no HURNP desde 1998 é o proposto por estudo que, 
partindo da necessidade de dimensionar pessoal e avaliar o trabalho de enfermagem em um hospital escola, validou um instrumento que classifica os pacientes em quatro graus ou níveis (I, II, III e IV) os quais representam, de maneira crescente, a dependência do paciente em relação à assistência de enfermagem. $O$ paciente é classificado dentro de um grau de acordo com a sua dependência da assistência de enfermagem nas atividades de: deambulação, banho, alimentação, comportamento, autocuidado, medicação, cuidados pré e pós-operatórios e manutenção da vida. Cada uma destas atividades possui características definidoras. Para que o paciente seja classificado dentro de um determinado grau, ele precisa apresentar no mínimo três características definidoras daquele $\operatorname{grau}^{(8)}$.

A classificação dos pacientes segundo ao grau de dependência de enfermagem é realizada diariamente, desde 1996, contemplando $100 \%$ dos pacientes internados na Instituição.

Os dados foram tabulados em planilhas e submetidos à análise com auxílio do Programa Microsoft Excel $2007 \AA$ e apresentados em forma de tabelas.
A coleta de dados somente se deu após aprovação do estudo pelo Comitê de Ética em Pesquisa da Universidade Estadual de Londrina, sob o parecer $n^{\circ}$ 182/09, e autorização formal da Diretoria de Enfermagem do HURNP.

\section{RESULTADOS E DISCUSSÃO}

Em 1998, o hospital em estudo tinha capacidade para 294 pacientes, destes 73 leitos estavam alocados na unidade Masculina, 11 leitos na Tisiologia e 46 na unidade Feminina. Dez anos depois, em 2008, com a implantação de novas unidades, o número de leitos da instituição aumentou para 317, porém, em relação às unidades de clínica médico-cirúrgicas alterou-se apenas o número de leitos na Unidade Masculina para 67. O dimensionamento de recursos humanos de enfermagem das referidas unidades, nos anos de 1998 e 2008, está demonstrado na Tabela 1.

Tabela 1: Quadro de recursos humanos de enfermagem das unidades de internamento médico-cirúrgicas adulto do HURNP, nos anos de 1998 e 2008, Londrina, PR, 2009.

\begin{tabular}{cccc}
\hline Categoria profissional & $\mathbf{1 9 9 8}$ & $\mathbf{2 0 0 8}$ \\
\hline Enfermeiros & 13 & 13 & 95 \\
& Técnicos / Auxiliares de enfermagem & 95 & 99 \\
\hline TOTAL & 108 & 112 \\
\hline
\end{tabular}

Fonte: Assessoria de Planejamento e Controle da Diretoria de Enfermagem HURNP, 2009.

Observa-se um aumento de 7,26\% em relação ao número de leitos ao longo dos anos, porém, no que diz respeito ao número de trabalhadores de enfermagem, este aumento não ultrapassou 3,57\%.

A adequação dos recursos humanos da instituição é uma tendência sem possibilidade de retrocesso, uma vez que os serviços de saúde caminham para a busca da melhora da qualidade no atendimento. Esta afirmação foi ratificada na investigação do dimensionamento de pessoal da equipe de enfermagem de todas as unidades do hospital estudado, que concluiu, anos mais tarde, que havia necessidade premente de ampliação do quadro de 15 e $20 \%$ para enfermeiros e técnicos/auxiliares de enfermagem, respectivamente. No mesmo estudo, os autores destacaram a defasagem do quadro de pessoal nas unidades de internação médico-cirúrgica e frisaram a necessidade de contratações para a área ${ }^{(9)}$.

Considerando a autonomia do enfermeiro nas unidades assistenciais para dimensionar e gerenciar o quadro de profissionais de enfermagem, o processo sofre influência direta de uma série de fatores determinantes, relacionados às questões institucionais. As Instituições públicas, como os Hospitais Universitários, possuem particularidades no que se refere ao vínculo empregatício que confere vários benefícios ao trabalhador, dentre os quais a estabilidade no emprego, além de uma legislação própria, característica dos servidores estatutários, que rege também a substituição de pessoal e aumento do quantitativo de profissionais ${ }^{(9)}$.

Neste contexto, é reconhecida a morosidade do processo de reposição da força de trabalho nas instituições públicas. Por isso, o Decreto $n^{\circ} 5.722$ de 2005, do Estado do Paraná, definiu a otimização de substituição de servidores lotados nos Hospitais Universitários nos casos de vacância de cargo decorrente de exonerações, aposentadorias e falecimentos, ficando as instituições autorizadas a realizar concursos públicos, com vista a evitar descontinuidade das atividades e, 
gradativamente, repor as vagas em aberto ${ }^{(10)}$. Mesmo com as melhorias, o processo permanece complexo e demorado, de modo que em 2008 ainda existiam 19 postos de trabalho desocupados, pertencentes à divisão de internamento do hospital, que se referem às unidades em estudo mais a unidade de moléstias infecciosas. Destas vagas em aberto, uma era de enfermeiro, duas de técnicos de enfermagem e 16 de auxiliares de enfermagem.

Os postos vagos refletem negativamente $e$ diretamente na qualidade da assistência prestada, bem como na área administrativa, ao aumentar o número de horas extraordinárias necessárias para prestar uma assistência segura; e também na saúde do servidor, devido à sobrecarga de trabalho.

O dimensionamento de recursos humanos para atendimento das necessidades de atenção de enfermagem aos pacientes é uma preocupação antiga de inúmeros pesquisadores, mas que permanece até os dias atuais $^{(2-3,9,11-13)}$. A inadequação de pessoal compromete seriamente a qualidade da assistência proporcionada à clientela, a sobrecarga de trabalho da equipe conduz ao estresse e, consequentemente, favorece a ocorrência de erros que podem causar sérios danos à saúde dos pacientes, com repercussões ético-legais para a equipe de enfermagem e para a instituição(11). Também é preocupante o aumento do absenteísmo não previsto, a falta de qualidade de vida no trabalho e a pouca satisfação dos profissionais de enfermagem ${ }^{(13)}$.

O hospital em estudo utiliza atualmente como referência para o dimensionamento do pessoal da enfermagem um referencial que propõe o cálculo das horas assistenciais de enfermagem necessárias para atendimento do paciente segundo sua clínica/especialidade ${ }^{(14)}$.

Considerando o número de funcionários demonstrado acima e o número de leitos quase inalterado entre os anos em estudo, verifica-se no cotidiano da enfermagem da instituição um aumento considerável do quantitativo de horas extraordinárias realizadas nas unidades de internamento masculino e feminino. A média mensal de horas extraordinárias realizadas no ano de 1998 na Unidade Masculina foi de 862 horas e 480 horas na Unidade Feminina. Em 2008, o quantitativo de horas realizadas cresceu para $1.159 \mathrm{e}$ 888 horas nas respectivas unidades. Este resultado poderia ser justificado pelo aumento do grau de dependência dos pacientes internados nos setores em estudo, ao longo desses 10 anos.

A crescente gravidade/dependência dos pacientes atendidos pode ser constatada no Gráfico 1, a seguir.

Gráfico 1: Total de paciente/dia atendidos nas unidades de internamento médico-cirúrgicas adulto do Hospital Universitário Regional do Norte do Paraná, segundo o grau de dependência da assistência de enfermagem, nos anos de 1998 e 2008. Londrina, PR, 2009.

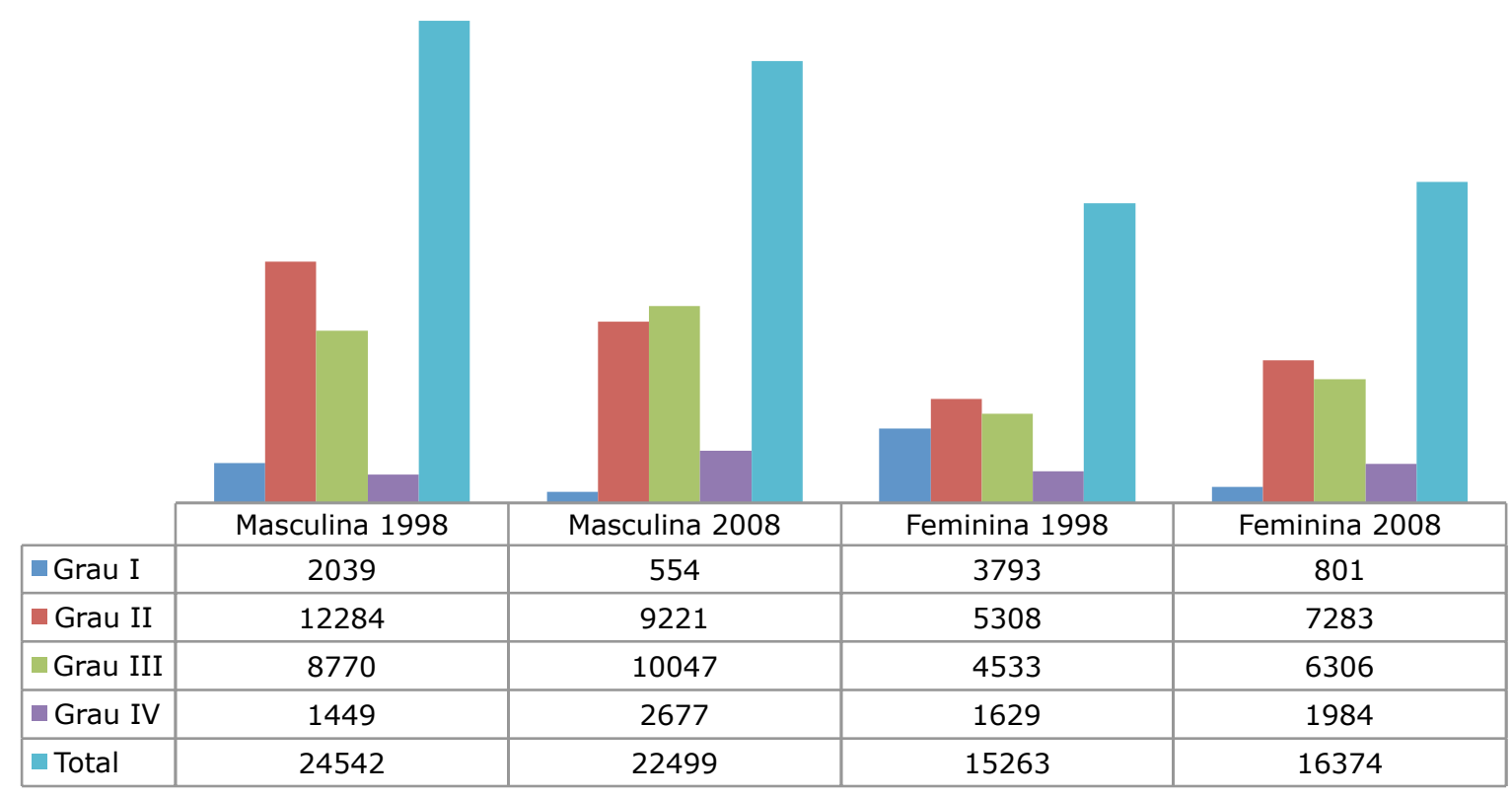

Fonte: Assessoria de Controle da Qualidade da Assistência de Enfermagem. HURNP, 2009. 
Verifica-se pelos dados do Gráfico 1 que em 1998 o número de paciente-dia na Unidade masculina foi 24.543 e na Unidade Feminina 15.263. Destes pacientes, aproximadamente $15 \%$ foram classificados como Grau I de dependência em relação à assistência de enfermagem, 44\% como Grau II, 33\% como Grau III e $8 \%$ como Grau IV.

No ano de 2008 , observa-se que o total de pacientedia na Unidade Masculina foi de 22.499 e na Unidade Feminina 16.374. Os percentuais de dependência da assistência de enfermagem no ano de 2008 foram assim distribuídos: 3\% Grau I, 42\% Grau II, 42\% Grau III e $12 \%$ Grau IV.

Utilizando outra metodologia, o estudo também classificou pacientes quanto ao grau de dependência de enfermagem em quatro níveis (cuidados mínimos, intermediários, semi-intensivos e intensivos) em unidades de internação médico-cirúrgicas em hospital universitário e identificou maior percentual de pacientes em cuidados intermediários, seguido de cuidados mínimos $^{(12)}$. Em outra pesquisa realizada, que utilizou instrumento de classificação com cinco categorias de cuidado, observou que $28,9 \%$ dos pacientes pertenciam à classificação de autocuidado, 41,9\%, intermediários, $22,1 \%$, alta-dependência, 5,8\%, semi-intensivo, e 1,3\%, intensivo(15).

Observa-se, portanto, no presente estudo, uma diferença significativa de pacientes que requerem maiores cuidados de enfermagem em unidades de internação no ano de 2008 em relação ao ano de 1998. Em relação ao demais estudos na área, seria inadequado tentar estabelecer uma relação entre eles em razão de que utilizaram instrumentos de SCP com metodologias distintas. Este aumento evidenciado nos pacientes classificados como grau III de dependência poderia ser explicado pela crescente demanda de pacientes com doenças crônico-degenerativas, geralmente classificados nesta categoria de dependência.

Neste contexto, os processos de transição demográfica e epidemiológica nacionais determinam importantes desafios para o sistema de saúde, como aumento desproporcional das faixas etárias mais elevadas, aumento da prevalência das doenças crônicas, aumento do número de indivíduos de alta dependência, número insuficiente de serviços especializados e maiores gastos com saúde ${ }^{(16)}$. O envelhecimento populacional tem levado a uma reorganização do sistema de saúde, pois essa população exige cuidados especiais, além do fato de que incorporam disfunções nos últimos anos de suas vidas ${ }^{(17)}$.

Além disso, foi verificado que mais de $10 \%$ dos pacientes internados nas unidades em estudo, no ano de 2008, foram classificados com Grau IV de dependência da enfermagem. Assim como nesse estudo, outra investigação também identificou a presença de pacientes em cuidados intensivos e semi-intensivos em unidades médico-cirúrgicas ${ }^{(12)}$. Os autores justificaram a permanência destas categorias de pacientes em unidade de internação em função da ausência de serviço de recuperação pós-anestésica no centro cirúrgico. No caso do hospital desse estudo, não se trata de carência de leitos de recuperação anestésica, mas sim, de leitos de UTI, pois sendo um hospital referenciado, a demanda por cuidados intensivos é alta e a oferta é insuficiente.

A presença de pacientes que necessitam de cuidados intensivos em unidades de internação, que não possuem aparato necessário para o atendimento e capacitação profissional adequada é cada vez maior. As características clínicas dos pacientes mais graves exigem da equipe de enfermagem atenção permanente e intervenções mais complexas, aumentando a carga de trabalho.

A falta de leitos intensivos para atender a toda clientela obriga os setores que não tem esta finalidade a absorver esta população. A mesma problemática foi encontrada em hospital do interior de São Paulo(18). A tendência de aumento do atendimento de pacientes de alta complexidade também está relacionada aos recursos tecnológicos utilizados no hospital e à grande variedade de especialidades médicas e de pesquisas clínicas ${ }^{(19)}$.

A caracterização do quadro de recursos humanos de enfermagem segundo a faixa etária apresentada na Tabela 2 evidencia o envelhecimento funcional e cronológico da força de trabalho em mais da metade da população estudada, a qual apresenta entre 40 a 59 anos. 
Tabela 2: Perfil do quadro de recursos humanos da Diretoria de Enfermagem do Hospital Universitário Regional do Norte do Paraná, segundo a faixa etária, nos anos de 1998 e 2008. Londrina, PR, 2009.

\begin{tabular}{|c|c|c|c|c|c|c|}
\hline \multirow{2}{*}{ Categoria profissional } & \multicolumn{2}{|c|}{ De 20 a 39 anos } & \multicolumn{2}{|c|}{ De 40 a 59 anos } & \multicolumn{2}{|c|}{ Acima de 60 anos } \\
\hline & 1998 & 2008 & 1998 & 2008 & 1998 & 2008 \\
\hline $\begin{array}{c}\text { Enfermeiros/ Técnicos/ Auxiliares/ Atendentes } \\
\text { de enfermagem }\end{array}$ & $30,25 \%$ & $33,15 \%$ & $19,25 \%$ & $62,7 \%$ & - & $4,15 \%$ \\
\hline
\end{tabular}

Fonte: Assessoria de Controle da Qualidade da Assistência de Enfermagem. HURNP, 2009.

Em se tratando de servidores públicos concursados, não se espera que o quadro de trabalhadores se renove frequentemente, como é comum se verificar em instituições de caráter privativo, pois, em função da estabilidade o servidor tende a permanecer na instituição até o final da carreira, ou seja, até a aposentadoria, e esta, muitas vezes compulsória.

Além disso, os trabalhadores dos serviços públicos do setor de saúde vêm sofrendo as consequências da redução do seu poder aquisitivo e buscando cada vez mais, principalmente na enfermagem, soluções como a adoção de outros vínculos empregatícios ${ }^{(20)}$, comprometendo ainda mais a sua saúde.

Ao envelhecimento da equipe, adiciona-se ainda a sobrecarga de trabalho, o alto grau de resistência física exigido, a exposição a doenças infecciosas e a carga psicológica vivenciada diariamente pelo profissional de enfermagem. Essa realidade evidencia a necessidade imediata de proporcionar melhores condições de trabalho à equipe de enfermagem, a fim de prevenir o adoecimento e perda precoce da capacidade para o trabalho.

\section{CONCLUSÃO}

Concluiu-se que o dimensionamento de pessoal de enfermagem não acompanhou o aumento da complexidade assistencial demandada pelos pacientes, 0 que é agravado pela capacidade para o trabalho reduzida dos trabalhadores.

\section{REFERÊNCIAS}

1. Kurcgant $P$, organizador. Gerenciamento em enfermagem. Rio de Janeiro: Guanabara Koogan; 2005.

2. Conselho Federal de Enfermagem. Resolução COFEN293/2004. Fixa e estabelece parâmetros para dimensionamento do quadro de profissionais de enfermagem nas unidades assistenciais das instituições de saúde e assemelhados [Internet]. Rio de Janeiro (Brasil): COFEN; 2004 [cited 2011 set 30]. Available from: http://site.portalcofen.gov.br/node/4329.

3. Campos LF, Melo MRAC. Dimensionamento de pessoal de enfermagem: parâmetros, facilidades e desafios. Cogitare Enferm [Internet]. 2009 [cited 2011 jul 30];14(2):237-46. Available
Os resultados também indicam a necessidade de imprimir esforços em busca de renovação e ampliação do quadro de trabalhadores de enfermagem do hospital. Além disso, é importante viabilizar soluções e mecanismos de gestão que respondam às dificuldades relacionadas à defasagem do quadro de pessoal, provendo horas de enfermagem para o atendimento seguro e de qualidade aos usuários dos serviços de enfermagem na instituição, assim como promovendo a redução da carga de trabalho aos servidores, já não tão jovens e, possivelmente com a saúde funcional comprometida em razão das características do trabalho em enfermagem.

Considerando que o dimensionamento do quadro de recursos humanos de enfermagem em instituições de saúde é imprescindível para a segurança do paciente, para a qualidade da assistência e, consequentemente, para a otimização da aplicação dos recursos em saúde, tendo em vista que pacientes mais bem tratados tem menores riscos de estarem expostos a eventos adversos e complicações, podemos citar Chenso( ${ }^{(9)}$ ao expressar que dimensionar recursos humanos em saúde vai mais além do que quantificar pessoas, envolve a qualificação/capacitação das mesmas para o cuidado.

Ressalta-se a importância da realização de novos estudos, a fim de obter subsídios para gestão hospitalar renovar e ampliar o quadro de trabalhadores de enfermagem do hospital, assim como comparar os resultados obtidos com hospitais de porte e complexidade semelhantes.

http://ojs.c3sl.ufpr.br/ojs2/index.php/cogitare/article/view/1560 9/10385.

4. Fugulin FMT, Gaidizinski RR. Dimensionamento da equipe de enfermagem em unidade de internação. In: Harada MJCS. Gestão em enfermagem. Ferramenta para a prática segura. São Caetano do Sul: Yendis Editora, 2011. p. 214-222.

5. Perroca MG, Gaidizinski RR. Análise da validação de constructo do instrumento de classificação de pacientes proposto por Perroca. Rev Lat Am Enfermagem [Internet]. 2004 [cited 2011 set 30];12(1):83-91. Available from: http://www.scielo.br/pdf/rlae/v12n1/v12n1a12.pdf.

6. Araújo VB, Perroca MG, Jericó MC. Variabilidade do grau de complexidade assistencial do paciente em relação à equipe de enfermagem. Rev Lat Am Enfermagem [Internet]. 2009 [cited 
2011 set 30];17(1): 34-39. Available from: http://www.scielo.br/pdf/rlae/v17n1/pt_06.pdf.

7. Martins PASF, Forcella HT. Sistema de classificação de pacientes na especialidade enfermagem psiquiátrica. Acta Paul Enferm [Internet]. 2006 [cited 2011 set 30];19(1):62-9. Available

from:

http://www.scielo.br/pdf/ape/v19n1/a10v19n1.pdf.

8. Martins EAP, Haddad MCL. Validação de um instrumento que classifica os pacientes em quatro graus de dependência da assistência de enfermagem. Rev Lat Am Enfermagem [Internet]. 2000 [cited 2011 set 30];8(2):74-82. Available from: http://www.scielo.br/pdf/rlae/v8n2/12421.pdf.

9. Chenzo MZB, Haddad MCL, Secco IAO, Dorigão AM, Nishiyama MN. Cálculo de pessoal de enfermagem em hospital universitário do Paraná: uma proposta de adequação. Semina. 2004;25(1):81-92.

10. Estado do Paraná. Decreto No 2755/2005 - Legislação Estadual de Recursos Humanos [Internet]. Curitiba (Brasil): Estado do Paraná; 2005 [cited 2011 set 30]. Available from: http://celepar7cta.pr.gov.br/seap/legrh-

v1.nsf/5199c876c8f027f603256ac5004b67da/d5443bfa79681ba2 832570cb005d65f3?OpenDocument.

11. Farias FAC. Dimensionamento de pessoal de enfermagem para pronto-socorro especializado em afecções cardiovasculares [thesis]. São Paulo: Escola de Enfermagem/USP; 2003. 257 p.

12. Nicola AL, Anselmi ML. Dimensionamento de pessoal de enfermagem em um hospital universitário. Rev Bras Enferm [Internet]. 2005 [cited 2011 set 30];58(2):186-190. Available from: http://www.scielo.br/pdf/reben/v58n2/a11.pdf.

13. Ohara R, Melo M R A C, Laus A M. Caracterização do perfil assistencial dos pacientes adultos de um pronto socorro. Rev Bras Enferm [Internet]. 2010 [cited 2011 set 30];63(5):749-54. Available from: http://www.scielo.br/pdf/reben/v63n5/09.pdf.

14. Saia A, Carro ARL, Corrêa AN, Coelho Junior C, Seixas PHD, Maeda ST. Parâmetros para o planejamento e dimensionamento da força de trabalho em hospitais gerais [Internet]. São Paulo: Secretaria de Estado da Saúde de São Paulo; 2006 [cited 2011 set 30]. Available from: http://www.observarh.org.br/observarh/repertorio/Repertorio_O bservaRH/SES-SP/Parametros_planejamento.pdf.

15. Carmona LMP, Évora YDM. Grau de dependência do paciente em relação à enfermagem: análise de prontuários. Rev Lat Am Enfermagem [Internet]. 2003 [cited 2011 set 30];11(4):468$473 . \quad$ Available from: http://www.scielo.br/pdf/rlae/v11n4/v11n4a09.pdf.

16. Areosa SVC, Areosa AL. Envelhecimento e dependência: desafios a serem enfrentados. Textos \& Contextos (Porto Alegre) [Internet]. 2008 [cited 2011 set 30];7(1):138-50. Available from:

http://revistaseletronicas.pucrs.br/ojs/index.php/fass/article/vie wFile/3943/3207.

17. Nasri F. O envelhecimento populacional no Brasil. Demografia e epidemiologia do envelhecimento. Einstein. 2008;6(1 Suppl):S4-S6.

18. Laus AM, Anselmi ML. Caracterização dos pacientes internados nas unidades médicas e cirúrgicas do HCFMRP-USP, segundo o grau de dependência em relação ao cuidado de enfermagem. Rev Lat Am Enfermagem. 2004;12(4):643-9.

19. Fakih FT, Carmagnani MIS, Cunha ICK. Dimensionamento de pessoal de enfermagem em um hospital de ensino. Rev Bras Enferm [Internet]. 2006 [cited 2011 set 30];59(2):183-7. Available from: http://www.scielo.br/pdf/reben/v59n2/a12.pdf. 20. Medeiros SM, Ribeiro LM, Fernandes SMBA, Veras VSD. Condições de trabalho e enfermagem: a transversalidade do sofrimento no cotidiano. Rev. Eletr. Enf. [Internet]. 2006 [cited 2011 set 30];8(2):233-40. Available from: http://www.fen.ufg.br/revista/revista8_2/v8n2a08.htm.

Artigo recebido em 06.12.2010.

Aprovado para publicação em 05.09.2011.

Artigo publicado em 30.09.2011. 